\title{
Science and ethics must not be separated
}

\section{The progress of research must be kept free from religious and political intervention.}

Sir - It is regrettable that ethics has been split from science and renamed bioethics. Ethics is an integral part of science. Like science, it requires us to be consistent and empirically justified in our interpretations of the actions of scientists. The ethics of science and science itself share the goal of comprehending in human terms scientists' actions in manipulating the physical world.

The division of science and ethics has been driven by an increasing interest in the actions of scientists by non-scientists. An unfortunate result of this has been a shift away from the consistent and justifiable methodology of science to an approach based on an often ill-defined 'personal philosophy' and 'gut feeling', for instance as described in Correspondence by D. P. Leader: "Reproductive cloning: an attack on human dignity" (Nature 424, 14; 2003).
Such 'gut feelings' undoubtedly play a part in science but are useless for proper understanding. The reactions of nonspecialist observers to complex ethical problems raised by cutting-edge science such as embryonic stem-cell research are no more justified or useful than their opinions about the technical difficulties yet to be overcome. The central issue in the ethical debate surrounding the embryo is not whether it is wrong to kill innocent human beings, but what the embryo and its disaggregation constitutes. The specialist scientific community's familiarity with the facts places it in a privileged position in determining the interpretation or interpretations best supported by the facts.

The rise of bioethics as an independent discipline has resulted in a confrontation between ethics and science that has obscured the similar aims of both. Of considerable concern is the increasingly political and religious nature of bioethics and the power it wields over the direction and progress of science. Science and the ethics of science are two sides of the same coin, dealing with the same empirical data and actions of the same scientists.

As well as thinking of their actions in terms of future experimental design, scientists must explain the significance of their actions in the wider scientific and human contexts. Scientists must take the lead in ensuring that the progress of science is both ethical and as free from political intervention as possible, if for no other reason than that only they can do so. Paul Copland

Department of Biochemistry, University of Otago, Box 56, Dunedin, New Zealand

\section{Safe home planned for lowa herbarium}

Sir - The plight of natural history collections is regrettable, as you report in the News story "Natural history collections in crisis as funding is slashed" (Nature 423, $575 ; 2003)$. You cite some examples of such collections in jeopardy, including the University of Iowa herbarium collection which is being moved to Iowa State University. In an era when state budgets are shrinking and debt is increasing, we feel that this cooperative venture is the most responsible way of managing and caring for a resource unique to the state.

The collections are not in jeopardy, as you suggest, but are being consolidated. As the state land-grant university, Iowa State University maintains active programmes in plant sciences, including an herbarium, while the University of Iowa has emphasized plant molecular biology.

The University of Iowa herbarium has not been a major research/teaching tool for many years. During 1998-2002, work at the Iowa State herbarium was cited in 75 publications, while work at the University of Iowa herbarium resulted in some 18 citations. Similarly, only two courses now use the herbarium at the University of Iowa, whereas herbarium use has remained steady at Iowa State. To ensure that the few faculty members at the University of Iowa using the resource have continued access, we will support travel between the two universities and have agreed that specimens needed for ongoing research may be kept on long-term loan.

Our intention is to retain all specimens that are not duplicated; the Iowa State herbarium has already applied for funds to accommodate the merged collections. If it becomes necessary to disperse some specimens, we will relocate them at institutions where they will be maintained and cared for. We began this process in the mid-1980s, when the bulk of the University of Iowa fungal collection was transferred to Iowa State University. Even if Iowa State cannot accommodate the entire remaining University of Iowa herbarium, it will keep all material collected in Iowa.

\section{Jack Lilien}

Department of Biological Sciences, University of Iowa, Iowa City, Iowa 52242-1324, USA

\section{Computer's ability to verify proof is an illusion}

Sir - Your news feature "Does the proof stack up?" (Nature 424, 12-13; 2003) addressing the difficulties faced by mathematicians in verifying the computer-aided putative proof of Kepler's conjecture concerning the densest arrangement of spheres - contains the seemingly reasonable statement by one mathematician: "I believe in a proof if I understand it". Yet it is well known that many a supposed understanding is a misunderstanding, and that there is no general rule guaranteed to distinguish between these opposite possibilities.

Arguments over the validity of mathematical proofs have raged for as long as mathematicians have been trying to prove conjectures. The history of this formal science is strewn with 'proofs' that have been accepted and later rejected, and 'proofs' that have been simultaneously accepted and rejected by different mathematicians.

The understanding of a computer program is no more reliable than the understanding of a mathematical proof.

Computers lure us into unmanageable complexity. It is, after all, one of their great assets that we can construct very long sequences of simple manipulations for a computer to execute at high speed and with unerring accuracy. However, this complexity (as with a list of alternative cases in a computer-aided proof) soon compounds, such that 'understanding' the program is impossible and a proof of the program is as complex as the program itself, or even more complex.

Scientists seek truths about the world (or about formal systems that mirror the world) and they do their best to confirm the validity of any new truth uncovered. But the reality is that (apart from certain limited totally abstract constructions) scientific truth can never be secure, validity can only be up to a certain point and formal verification is not a practical option. Scientific truths are best-guesses given all the available evidence, or, more pessimistically, they are potential errors as yet unmasked. In sum, computers add nothing new to the difficulties of proof and verification - they simply enlarge existing problems through the magnifying glass of complexity escalation.

Derek Partridge

Department of Computer Science,

Harrison Building, University of Exeter,

Exeter EX4 4QF, UK 